Supplement of Saf. Nucl. Waste Disposal, 1, 303-303, 2021

https://doi.org/10.5194/sand-1-303-2021-supplement

(c) Author(s) 2021. CC BY 4.0 License.

Supplement of

\title{
What do we need to trust in models?
}

Ingo Kock et al.

Correspondence to: Ingo Kock (ingo.kock@bfe.bund.de)

The copyright of individual parts of the supplement might differ from the article licence. 


\section{Beyond good practices?}

\section{Building confidence in modelling results}

Federal Office

for the Safety of

Nuclear Waste Management

Contribution to workshop II

"What do we need to trust in models? “

Martin Navarro, BASE 


\section{Good practices help us in building confidence in modelling results}

- Methods for uncertainty analysis: FEP-catalogues, scenario development, alternative or complementary models, parameter variation, sensitivity analysis, ...

- Quality assurance of data, codes and calculations: clearance processes, verification tests, benchmarks, use of alternative or complementary models, validation where possible, ...

- Management processes: preparing input data and keeping them consistent, planning and executing calculations, interpreting and documenting results, ...

- systematic

- traceable

- transparent

- quality-assuring

- help to justify selection of models and calculation cases

and more 


\section{Beyond good practices}

Establishing good practices in an organisation is the first thing to do.

However, good practices or systematic approaches are not the solution to everything. 


\section{Three inconspicuous modelling challenges}

... which probably can not be solved by systematic approaches alone

... which we usually do not talk about

... which probably should receive more attention

Two technical issues

1. Justification of enforced simplifications

2. Investigation of model uncertainties

On non-technical issue

3. Showing the adequacy of models in face of the validation problem 
\#1. Justifying enforced simplifications 


\section{Model always simplify}

Many simplifications are not deliberate but

technically enforced by features and limitations of codes

"we have to do it that way with this code"

"that's how it is usually done"

"we have tried to keep it simple"

These are no scientific justifications!
2D, simple geometries, homogenisation, no scale dependencies, no localizing processes, transferability of features to other locations or scales, Darcy's law applicable, concept of intrinsic permeability valid, only one species per radionuclide (transport, sorption), no process coupling, standard curves for Pcap or Krel apply, initial hydraulic equilibria, equilibrium density distribution in saline aquifers, standard hydraulic boundary conditions apply, hydraulic boundaries exist, planes of symmetry, boundary conditions don't change with time.

Discrete fracture networks:

Evolution of joint networks is similar to a stochastical process, independent generation of joints, joints near to planar, simple internal joint structure, simple joint intersections. 


\section{A justification gap}

If simplifications are technically enforced

a scientific justification is not automatically at hand!

Sometimes we may be at a loss:

- Because there is no scientific justification

- Because scientific justifications are far fetched

- Because further investigations exceed the given resources

$\rightarrow$ Increased likelihood that enforced simplifications lack scientific justification 


\section{What can we do?}

- Try not to underestimate the resources needed to justify "simple" models

- Keep an eye on enforced simplifications in model reviews

Is this good practice?

Could it become good practice? 
\#2. Investigating model uncertainties 


\section{Systematic treatment of model uncertainties?}

Good practices exist for scenario and parameter uncertainties

Author not aware of systematic approaches for the investigation of model uncertainties.

Practical modelling shows that there are at least technical reasons for this.

Model uncertainties

= uncertainties arising

"[...] from an incomplete knowledge or lack of understanding of the behaviour of natural and engineered systems, physical processes, site characteristics and their representation [...]"

(MeSa 2012) 


\section{Investigating alternative (sub-)models}

Let's assume you would like to investigate a specific alternative process model.

What would you do ...

- ... if the process is not implemented in your code or in any other code available in your organization?

- ... if that means that you have to repeat all parameter variations for all combinations of alternative sub-models?

There is an increased likelihood that the investigation of model uncertainties is incomplete 


\section{What can we do?}

- Do not underestimate the resources to investigate alternative models

- Keep an eye on the investigation of alternative models in model reviews

Again:

Is this good practice?

Could it become good practice? 
\#3. Showing the adequacy of models 


\section{Showing model adequacy}

Models are adequate if they tell us something about the real repository system and its safety

The central question: How can we exclude that we have missed something important in the construction of our models?

The standard answer: validate! (check against reality)

The problem: Models for long-term processes cannot be validated!

(NEA 1991, MeSa 2012) 


\section{The validation problem}

Why we can't validate

- Timescales beyond human experience

- Conservative models do not aim at representing reality

- Most scenarios will never become reality

\section{Our general assessment strategy}

We do not check our models against reality but we collect arguments why we believe that our models are adequate

= We don't show that we hit the target but that we aim well In this framework we have to be aware of 2 epistemic pitfalls that can mislead us 


\section{Pitfall 1: We tend to see what we expect}

Norwood Russell Hanson: Theory-ladenness of observation

We cannot look at the world without interpreting it in terms of theories.

We look at repository systems with certain expectations or models in mind 


\section{Pitfall 1: We tend to see what we expect}

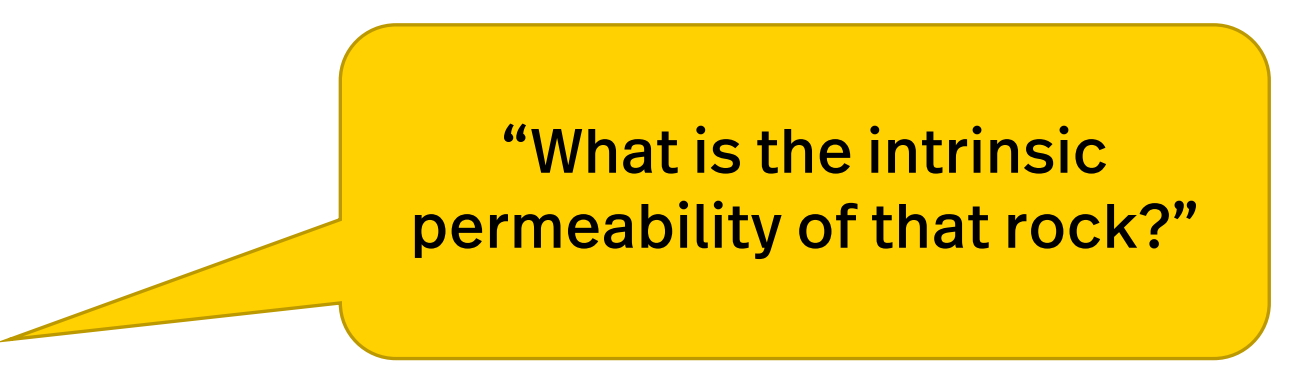

"What is the size statistics of this joint network?"

\section{Expectations}

- We are dealing with laminar flow in a porous medium

- The theory of a phase-independent, intrinsic permeability is empirically adequate.

- The considered rock volume has a representative permeability (e.g. no scale dependency)

- Joints are approx. 2D structures with measurable size (e. g. no complicated Riedel shear systems)

- The network mostly consists of joints 


\section{Pitfall 1: We tend to see what we expect}

The danger

It is possible that we think we are observing the repository system

when we are in fact only observing a subjective image of the system

As modelers we learn to think as our codes do. That creates expectations. It increases the likelihood that we find our model applicable.

If you have a hammer everything you see is a nail. 


\section{Pitfall 1: We tend to see what we expect}

\section{What we can do}

- Identify and question expectations

- Widen expectations by research, experience and by making mistakes

Difficult to tackle in a systematic way 


\section{Pitfall 2: Believing that a model is confirmed although it is not}

The holoistic Duhem-Quine thesis

Scientific hypotheses or theories are underdetermined by empirical data

If our model matches empirical evidence ...

- Other models might match them too

- We might rely on false background beliefs

E. g. "I can see all relevant effects in this experiment"

There is no final model confirmation.

We have to think about the nature of model confirmation. 


\section{Pitfall 2: Believing that a model is confirmed although it is not}

What we can do

- Revisit models even if they seem to be confirmed

- Become aware of background beliefs and question them

Again, there are no systematic approaches for this. 


\section{What do we need to trust in models ...}

... in face of the aforementioned problems? 


\section{If we can't find suitable systematic approaches}

\section{Question models and modelling results! Try to falsify!}

- The scientist's confidence in model results increases with every failed attempt of falsification.

- Questioning is what makes our models scientific models.

$\rightarrow$ Confidence in modelling results does not only need good practices but also a devil's advocate! 